\title{
ESTÁNDAR OPC UA COMO SOLUCIÓN A LA INTEGRACIÓN INDUSTRIAL EN UNA EMPRESA
}

\section{OPC UA Standard, a Solution to Industrial Integration, Case Productos del Trópico, S.A.S.}

\author{
José ANdrickson ${ }^{a}$, EnRique Gabriel ${ }^{b}$, Edgar ChacóN ${ }^{c}$, \\ Alama Puello ${ }^{d}$, Félix Rondon ${ }^{e}$, Wander Emeterio ${ }^{f}$ \\ y Montgomery Reyes ${ }^{g}$
}

Recibido: 8/7/2021 • Aprobado: 2/10/2021

Cómo citar: Andrickson, J., Gabriel, E., Chacón, E., Puello, A., Rondon, F., Emeterio, W., \& Reyes, M. (2021). Estándar OPC UA como solución a la integración industrial en una empresa. Ciencia, Ingenierías y Aplicaciones, 4(2), 41-65. Doi: https://doi.org/10.22206/cyap.2021.v4i2.pp41-65

\section{Resumen}

En el diseño de la arquitectura de software y la toma de decisiones dinámica existen factores importantes para la obtención de sistemas flexibles y escalables de alto rendimiento económico. Este proceso exige planificación, selección y el uso recurrente de los sistemas de información que permitan solventar los cambios externos e internos presentes en las empresas. La investigación demuestra que los entornos dinámicos e integrados son enfoques para abordar las arquitecturas de información de sistemas modernos de producción; en este sentido, es necesario diseñar e implementar modelos de integración que incorporen

\footnotetext{
a Instituto Especializado de Estudios Superiores Loyola, Facultad de Ingeniería, Dirección de Investigación, San Cristóbal, República Dominicana. Univerlsidad de Los Andes, Facultad de Ingeniera, Mérida, Venezuela. Univerlsidad Nacional Experimental del Táchira, Decanato de Investigación, Táchira, Venezuela. ORCID: 0000-0003-4026-4548

b Instituto Especializado de Estudios Superiores Loyola, Facultad de Ingeniería, Dirección de Investigación. Correo-e: enriquegabrielcarmona@gmail.com

c Univerlsidad de Los Andes, Facultad de Ingeniera Laboratorio de Sistemas Discretos, Automatización e Integración, Mérida, Venezuela. ORCID: 0000-0001-5612-0368

Correo-e: echancon@ula.ve

d Instituto Especializado de Estudios Superiores Loyola. Correo-e: luceropuello@outlook.com

${ }^{\mathrm{e}}$ Instituto Especializado de Estudios Superiores Loyola. ORCID: 0000-0001-8022-6701

Correo-e: frondon@ipl.edu.do

${ }_{\mathrm{f}}^{\mathrm{f}}$ Instituto Especializado de Estudios Superiores Loyola. Correo-e: wanderemeterio@gmail.com

g Grupo de empresa Corripio, Productos del Trópico, Administración. San Cristóbal, Republica Dominicana. Correo-e: mreyes@corripio.com.do
}

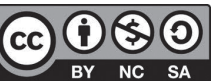


la reconfiguración dinámica con una filosofía de plataforma abierta, que en la actualidad son considerados para el diseño de soluciones empresariales. De esta manera esta investigación plantea el desarrollo de una arquitectura de información basada en el estándar OPC UA para la integración de la información en una empresa, lo cual permite simplificar los procesos de toma de decisiones.

Palabras clave: industria; arquitectura de información; modelo; sistemas de información; OPC UA.

\begin{abstract}
VIn software architecture design and dynamic decision making there are important factors for obtaining flexible and scalable systems with high economic performance. This process requires planning, selection and the recurrent use of information systems that allow solving the external and internal changes present in companies. Research shows that dynamic and integrated environments are approaches to address the information architectures of modern production systems; In this sense, it is necessary to design and implement integration models that incorporate dynamic reconfiguration with an open platform philosophy, which are currently considered for the design of business solutions. In this way, this research proposes the development of an information architecture based on the OPC UA standard for the integration of information in a company, which allows to simplify decision-making processes.
\end{abstract}

Keywords: industry; information architecture; model; information systems; OPC UA. 


\section{Introducción}

La empresa Productos del Trópico, ubicada en San Cristóbal, República Dominicana, tiene como propósito la producción y exportación de bebidas y alimentos enlatados, cuya calidad ha sido reconocida internacionalmente en reiteradas oportunidades (Ruiz, 2019). Adicional a su solidez económica y corporativa, esta entidad se ha caracterizado por su responsabilidad social (Molina, 2019). En los últimos ańos la empresa ha incorporado en sus 13 procesos productivos un total de 247 equipos de automatización industrial con la finalidad de optimizar tales procesos (Garay, 2020). Estos equipos de automatización utilizan cinco protocolos de comunicación diferentes, distribuidos entre los equipos industriales de la siguiente manera: el $66.6 \%$ vía Serial, el $9.5 \%$ vía Ethernet IP, el $9.5 \%$ vía Profinet, el $9.5 \%$ vía Profibus y el $4.9 \%$ vía USB. Dado que no todos los equipos industriales utilizan el mismo protocolo de comunicación, actualmente los procesos productivos no se pueden gestionar de manera conjunta y centralizada, trayendo como consecuencia islas de información, que generan inconvenientes en el proceso de creación, extracción y auditoría de datos ubicados en las líneas de producción.

En vista de que actualmente la capacidad de compartir información desde, entre y hacia las líneas de producción no es posible dada la carencia de una arquitectura unificada (cliente-servidor) capaz de propiciar esta integración e interoperabilidad industrial, se deben realizar reportes manuales que contengan estos datos de interés, los cuales son propensos a errores humanos y del mismo modo afectan ampliamente la integridad de la información. Para validar las informaciones contenidas en estos reportes, la empresa necesita detener las líneas de producción y realizar auditorías periódicas, que imposibilitan el flujo continuo de los procesos productivos, generando una merma en los beneficios económicos para la empresa. Del mismo modo, esto impacta en las estadísticas consideradas para definir el curso de acción de la empresa.

Actualmente, es difícil acceder a datos de las líneas de producción en tiempo real, ni desde cualquier lugar, limitando la capacidad de integrar al sistema de gestión de información corporativo con las líneas de producción, lo cual añade complejidad al sistema productivo y genera 
mayores inversiones y hace costosa cualquier modificación en materia de optimización de la empresa. Basados en estas premisas, es necesario analizar indicadores técnicos y económicos que permitan medir la factibilidad de la implementación de una arquitectura unificada, con aceptación y estandarización internacional, apta para garantizar el flujo de información entre elementos de automatización industrial diseñados por diferentes entidades y también hacia los sistemas de gestión de información corporativos.

\section{Descripción del sistema}

Para un manejo eficiente y productivo, las empresas frecuentan la gestión eficiente de los equipos y simplificación de sus procesos, más aún cuando se utilizan equipos y sistema distribuido de manera funcional y/o geográfica. El mayor problema de compartir información entre equipos, sistemas de control y los recursos es la heterogeneidad de los fabricantes de soluciones, es por ello que la falta de interoperabilidad o integración trae consigo las islas de información.

Con el propósito de solventar estos problemas, en 1996 fue creado el estándar Open Platform Communications (OPC, por su sigla en inglés) y alrededor del ańo 2012 ya existían aproximadamente 50 millones de equipos industriales con la tecnología OPC implementada, de los cuales el 43.9 \% eran Controladores Lógicos Programables (PLC, por su sigla en inglés), el $32.1 \%$ son operadores de panel, el $14.5 \%$ son Interfaz hombre-máquina (HMI, por su sigla en inglés) y el $9.3 \%$ son Computadoras Personales Industriales (IPC, por su sigla en inglés) (Resnick \& Clayton, 2018).

En Productos del Trópico S.A.S. este panorama es una situación común, por lo cual la empresa se ve obligada a detener sus procesos productivos periódicamente, casi el $7 \%$ del ciclo anual, para validar la fiabilidad de las informaciones suministradas por las referidas islas de información, afectando principalmente su rentabilidad económica y, a la vez, exponiendo los procesos a amenazas internas y/o externas referentes a la integridad y seguridad de la información. 


\subsection{Consideraciones del proyecto}

Al incorporar el estándar OPC UA en las operaciones de Productos del Trópico S.A.S., se podrá lograr la interoperabilidad de los 247 equipos industriales que intercambiarán la información de manera bidireccional sin la necesidad de interfaces de comunicación particulares e inherentes a cada fabricante. En consecuencia, la transmisión/recepción de información entre todos estos elementos de automatización y las 13 líneas de producción será efectiva, ágil, rápida y segura, por consiguiente, los inconvenientes de integración de la información no existirán.

Las tecnologías OPC UA se crearon para permitir que la información se gestione y se intercambie de forma eficiente entre diversas plataformas de múltiples proveedores, lo cual permite un manejo superior a los procesos manuales que la empresa está enfrentando en la actualidad. A su vez, se diseñaron para asegurar una integración perfecta entre plataformas sin necesidad de un desarrollo de software costoso y usualmente limitado. Tal solución satisface de manera específica las necesidades en materia de automatización y redes de los procesos productivos de Productos del Trópico S.A.S, haciendo que los mismos estén preparados para los retos del presente y del futuro.

\section{Contexto del proyecto}

Productos del Trópico S.A.S. inicia sus operaciones comerciales en el año 1971, desde San Cristóbal, República Dominicana. Esta pertenece a la categoría de 'Zona Franca Especial' debido a que se dedica al procesamiento y la exportación de productos alimenticios, tales como: leche, habichuelas, jugos o néctares, crema de coco y pasta de tomate. Del mismo modo, cabe resaltar que dicha entidad forma parte del Grupo Corripio. Según Ruiz (2019), en su trayectoria comercial la compañía se ha caracterizado por diversos factores, dentro de los cuales se destaca su política alimentaria, cuyo enfoque fundamental es exceder los requisitos de sus clientes al suministrarles productos inocuos y consistentes que garanticen la excelencia mediante la mejora continua. Todo esto con el objetivo de que los clientes reciban una calidad de clase mundial. 
Se ha evidenciado un crecimiento sostenible en la empresa, donde todas sus líneas de producción han sido ampliadas, trayendo la readecuación de la infraestructura de automatización y también la arquitectura de redes y comunicaciones. Dicha mejora genera un impacto directo en su posicionamiento corporativo a nivel nacional e internacional y también en las responsabilidades diarias de sus colaboradores. Estos cambios motivaron a incluir en sus respectivas infraestructuras nuevos elementos para ayudar a perfeccionar el flujo de información y también el control de acciones puntuales en las diferentes líneas de producción. Diversos levantamientos de información dieron como diagnóstico inicial la presencia de una gran cantidad de procesos manuales.

Los procesos de integración generan oportunidades de mejora en aspectos relacionados a los Sistemas de Planificación de Recursos Empresariales (ERP, por su sigla en inglés), Manejos de Ejecución de Sistemas (MES, por su sigla en inglés), puesta en marcha de seguridad informática, respaldo de bases de datos, modelado de información y de igual manera la interoperabilidad con otros elementos de automatización.

Como propuesta a mejorar la eficiencia y rendimiento en la empresa, se desarrollaron implementaciones a través de la incorporación de PLC de diversas marcas con transmisión de información de diferentes protocolos de comunicación industrial, algunos convencionales, otros en etapa de evaluación. Pese al avance tecnológico incorporado, la etapa de gestión y monitoreo de procesos generó diferentes desafíos; por ejemplo, las marcas HMI, utilizadas en la empresa, no permitían ser integradas con todos los elementos de automatización, impactando así la toma de decisiones.

Quedó de manifiesto el hecho de que varios equipos de la empresa debían ser tratados como elementos aislados de información por la carencia de una infraestructura que permitiese la integración industrial. En ese mismo orden, también se hacía complejo asegurar la información y los cortafuegos (en lo adelante, firewalls) capaces de monitorear y resguardar el tráfico generado por los diferentes equipos industriales y proveniente de algún cliente informático hacia los equipos industriales ya mencionados. Añadiendo complejidad a la problemática, la referida situación no permitía 
conexiones remotas a estos dispositivos industriales o integración a los elementos activos de la red de la empresa (Routers, servidores, Switches, etc.). En resumen, existe la necesidad de integración de la línea de producción a un único sistema de gestión información.

La integración industrial y la gestión centralizada son elementos claves para afrontar los desafíos de crecimiento y calidad de la empresa. Sin este cambio de arquitectura, la empresa no puede valerse de las bondades de la Industria 4.0 y sus recursos (Realidad Aumentada, Big Data, Deep Learning, Gemelos Digitales, Calidad 4.0, Cloud Computing, Smart Grids, entre otros).

La gerencia general, en conjunto con el personal a cargo de las operaciones críticas de la corporación, desea enriquecer y ampliar los métodos para el manejo de la información e integración industrial de los subsistemas de Productos del Trópico S.A.S. Para esto, algunos objetivos buscados son:

- Estadísticas de las líneas de producción.

- Histórico digitalizado de la operatividad de los equipos industriales.

- Monitoreo centralizado de eventos.

- Aseguramiento de la confidencialidad, disponibilidad e integridad de la información.

- Integración con clientes informáticos.

Pese a toda la situación expuesta anteriormente, los procesos productivos de la empresa marchan sin mayores inconvenientes. De manera constante se incorporan nuevas tecnologías que aportan eficiencia a los mismos. Sin embargo, para lograr la adecuación de la infraestructura de automatización y las de redes y comunicaciones, que utilicen elementos de Industria 4.0 y, por su puesto, estandarización e integración industrial, Productos del Trópico S.A.S., necesita un rediseño en la arquitectura de información, por lo cual se decidió abordar un estudio piloto de los procesos de producción de Pasta de Tomate (línea 3). 


\section{Antecedentes}

La implementación de la arquitectura de información inicia con evaluar el caso de estudio, además de una revisión bibliográfica de las posibles soluciones. Una vez seleccionado un paradigma que plantee una solución factible, se realiza una revisión de algunos estudios que soporten la propuesta, en este caso podemos referenciar:

- Barnstedt (2019), quien define a OPC UA como la base esencial para la convergencia de la tecnología operacional y las tecnologías de la información, ya que proporciona el modelado y estandarizado de los datos para los equipos industriales. El protocolo sirve como tecnología de puerta de enlace para habilitar de forma segura equipos industriales en la nube, permitiendo así la gestión de datos y dispositivos, además del aprendizaje de máquinas que no fueron diseñadas para tener estas capacidades.

- EU Automation (2019) establece que las pequeñas y medianas empresas a raíz del estándar OPC UA se han beneficiado al contar con una conectividad total en sus procesos de producción. Dicho estándar, comunica los PLCs de diferentes proveedores y también permite la interconexión de SCADA, MES y ERP, para dar al cliente una visión completa de la empresa.

- INTRAVIS (2017), realizó una investigación con simulación, cuyo objetivo era producir una tapa de agua, luego influenciar externamente la máquina y a través de una interfaz del estándar OPC UA se le informa a la máquina de moldeo de inyección para ajuste los parámetros, y así en un tiempo corto, la máquina vuelve a producir, evitando detener la producción y generar gastos.

- En Felt (2019), varias empresas dieron referencias de cómo la integración de OPC UA mejoró sus procesos industriales. Dentro de estas se encuentra AREVA, la cual, desde temprano, reconoció el potencial de OPC UA, por consiguiente, comenzaron a integrarlos en instrumentos de monitoreo (SIPLUG) y los utilizaban para monitorear sistemas críticos en entornos remotos. Lo cual facilitó 
varios aspectos como el almacenamiento de datos o análisis de datos. Aquí se explica cómo OPC UA permite la incrustación directa de los productos en la infraestructura.

\subsection{Características de OPC UA}

OPC UA es una plataforma abierta de múltiples conexiones a dispositivos de campos y de integración de sistemas. Para comunicar se tiene un OPC UA Cliente y OPC UA Servidor, ambos cumplen funciones específicas para la conexión de la información de un sistema.

- OPC UA Cliente: está compuesto por una interfaz de usuario que admite los principales modelos de información de la arquitectura unificada de OPC. Entre estos modelos ofrecen acceso a datos, alarmas y condiciones y acceso a datos históricos. Posee una mejorada interfaz de usuario para leer y escribir datos directamente al servidor OPC, con mayor seguridad.

- OPC UA Servidor: es un equipo utilizado para alojar servicios u otras especificaciones para que ciertas operaciones puedan realizarse mediante procesos determinados por un administrador. Para que un servidor sea OPC debe tener todas las especificaciones marcadas por OPC Foundation.

Entre otras características de OPC UA tenemos:

- Interconexión con distintas fuentes de información en diferentes entornos de producción y fabricación como empresas, laboratorios, entre otros.

- Capacidad multiplataforma que elimina la dependencia de un sistema operativo.

- Seguridad expandida y certificados

- Integración de tecnología de la información

- Combatividad con versiones anteriores 
- Conexión de aplicaciones, basado en una tecnología web

- Implementación, donde se proporciona componentes de hardware y software, dispositivos y sistemas automatizados.

- Escalabilidad a través del desarrollo de aplicaciones clientes de OPC.

- Eliminación de bloques de conexiones, ya que se centra en el desarrollo de funcionalidad de aplicación.

- Esquema abierto, donde los usuarios finales pueden elegir entre hardware y software de diferentes proveedores, donde los componentes deben funcionar perfectamente.

- Acceso a datos relacionados con proceso en todos los niveles de empresa.

- Uso de etiquetas configurada por el usuario, dado que el servidor puede automatizar el proceso.

- Resolución de problema, reduciendo la necesidad de ser experto en cada protocolo.

- Agregar/eliminar, sin apagar el sistema, elementos, y cuando se hace una solicitud por el cliente, los datos se remiten vía servidor al momento del requerimiento.

- No obsolescencia, dada la tecnología de COM, DCOM, ActiveX

\section{Ciclo de producción y manejo de información actual de la empresa}

Productos del Trópico S.A.S. actualmente cuenta con trece (13) líneas de producción destinadas a la creación y/o procesamiento de diferentes productos, como son: néctares, jugos, habichuelas, pasta de tomate y derivados del coco. A modo general, dichas líneas de producción se subdividen en procesos similares. Dado el interés de analizar la factibilidad de una arquitectura unificada (cliente-servidor), se tomará como caso de estudio la línea tres (3), cuyo propósito es la fabricación de pasta de tomate (Ruiz, 2019). Acorde a la figura 1 se muestra el diagrama de flujo de pasta de tomate, el proceso se compone de diez (10) subprocesos y 
cada uno de estos posee funciones puntuales, las cuales, a grandes rasgos, necesitan de intervención humana y/o artificial mediante diferentes elementos de automatización industrial. Tales subprocesos generan información relevante para la continuación de las etapas posteriores, sin embargo, la consulta y/o transmisión de dichas informaciones tiene un importante componente manual que afecta su manejo.

\section{Figura 1}

Diagrama de flujo del proceso Pasta de Tomate

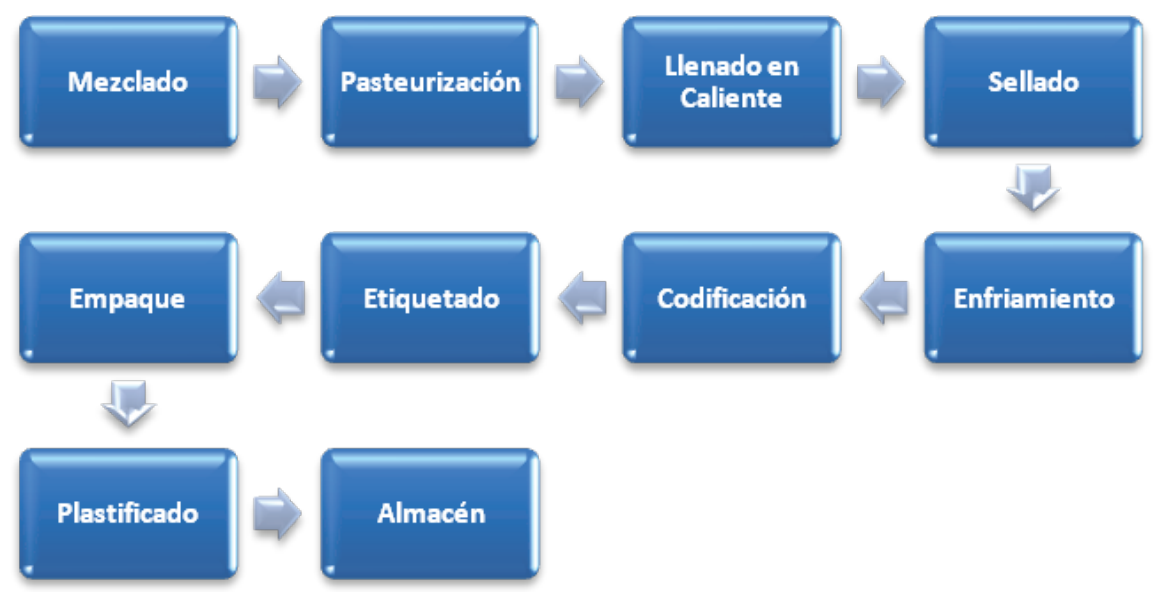

Según el escenario, los subprocesos de etiquetado, empaque, plastificado y almacén pueden gestionarse informáticamente de la misma manera. Tal y como se ha observado, el ciclo de vida del producto tiene un orden lógico y funcional, donde el aspecto más crítico de la logística es la toma de decisiones operativas y administrativas basada en la información recolectada e ingresada de forma manual. En este sentido, el manejo de la información provista por la línea 3 es vulnerable y susceptible a errores humanos, y no puede ser gestionada de forma digital, de manera remota. Por esta razón, se hace necesario incorporar al sistema de gestión de información un elemento interfaces entre la arquitectura de automatización y el sistema de gestión. Comparando este panorama con los resultados obtenidos por Bańo (2019), se puede confirmar que OPC UA permite lograr la interconexión deseada para afrontar los inconvenientes planteados. 


\subsection{Propuesta para la implementación de OPC UA}

Para la implementación de la arquitectura de información basada en OPC UA se desarrolló un estudio aplicativo a través del software Prosys (Prosys OPC, 2019), con conocimiento a fondo del estándar OPC UA, destacando las capas del Stack de dicha tecnología. El Stack hace referencia a los componentes de software utilizados por otras aplicaciones para la implementación del estándar. Está conformado por: codificación de la información, seguridad de la información y el transporte de los datos.

\section{Codificación de los datos en OPC UA}

Para la codificación de datos se definen la serialización de parámetros de servicio en formato binario y XML (Sagñay, 2016). Estos generalmente se transmiten en una secuencia de bytes. El estándar OPC UA define dos tipos de codificación de datos: Binario OPC UA y UA XML, como se muestra en la figura 2 del servidor Prosys (Prosys OPC, 2019), en Productos del Trópico S.A.S.

\section{Figura 2}

Binario OPC UA y UA XML

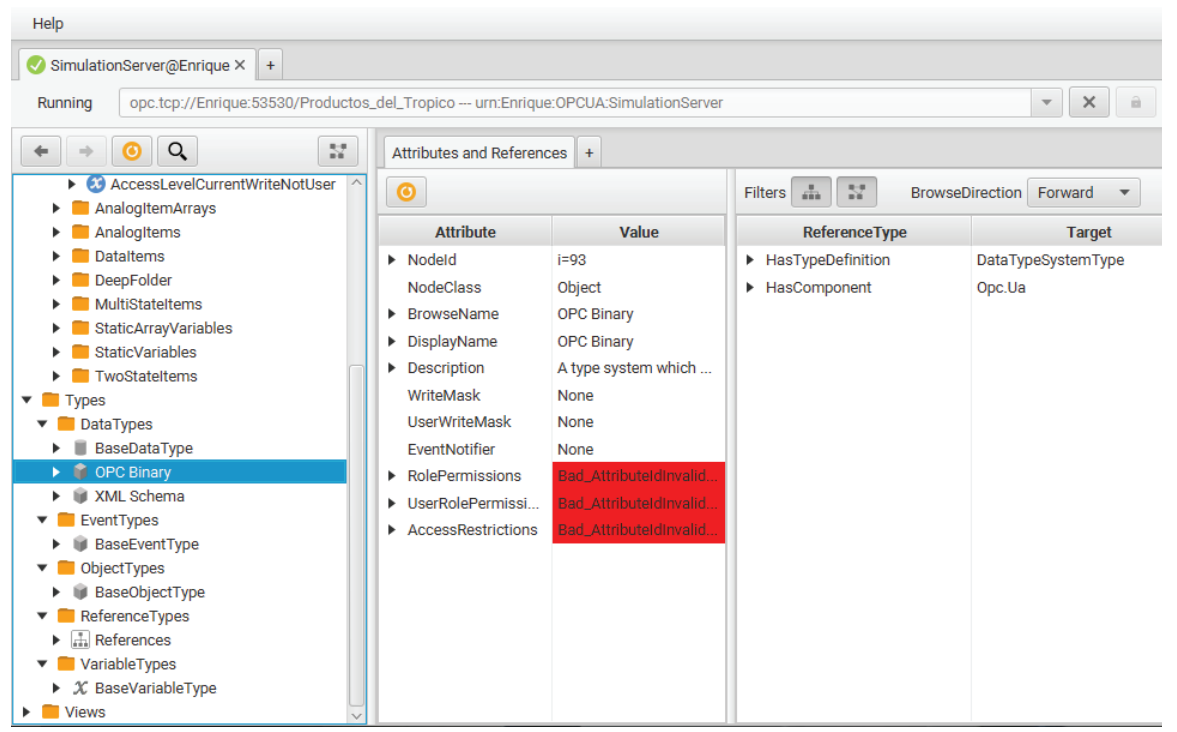




\section{Protocolo de seguridad en OPC UA}

Todas las aplicaciones para comunicarse a través de la tecnología OPC UA deben crear un canal seguro (SecureChannel) y establecer una sesión (session) antes de consumir cualquier servicio implementado, esto es conocido como una Conversación Segura (Security Handshake). Los protocolos de seguridad pueden soportar tres modos de seguridad: Ninguno (None), Firmado (Sign), y Firmado y cifrado (SignAndEncrypt) (Sagñay, 2016). Tales opciones están disponibles en el servidor Prosys (Prosys OPC, 2019), que fue utilizado para emular funcionalidades en Productos del Trópico S.A.S. El set de algoritmos de seguridad utilizados durante una conversación segura es llamado la política de seguridad (Security Policies). Las aplicaciones deben especificar la política de seguridad mediante un URI (Uniform Resource Identifier), el cual es un conjunto de caracteres que identifica un recurso en red en Productos del Trópico S.A.S.

La figura 3 ofrece una visión general sobre las asignaciones definidas, teniendo en cuenta las soluciones de seguridad. En esta se muestran los protocolos de transporte (UA TCP Y SOAP), los protocolos de seguridad (Conversación Segura UA y Conversación Segura WS) y los puertos utilizados por cada protocolo. El buffer mínimo que requiere la conversación segura OPC UA es de 8196 bytes. Todas las seguridades son aplicadas a cada pedazo y no al mensaje entero. El Stack debe tener implementado la aplicación de seguridades a cada MessageChunk y la habilidad de recibir los pedazos y ensamblar al mensaje completo. 


\section{Figura 3}

Protocolos de transporte, seguridad y cifrado en OPC UA

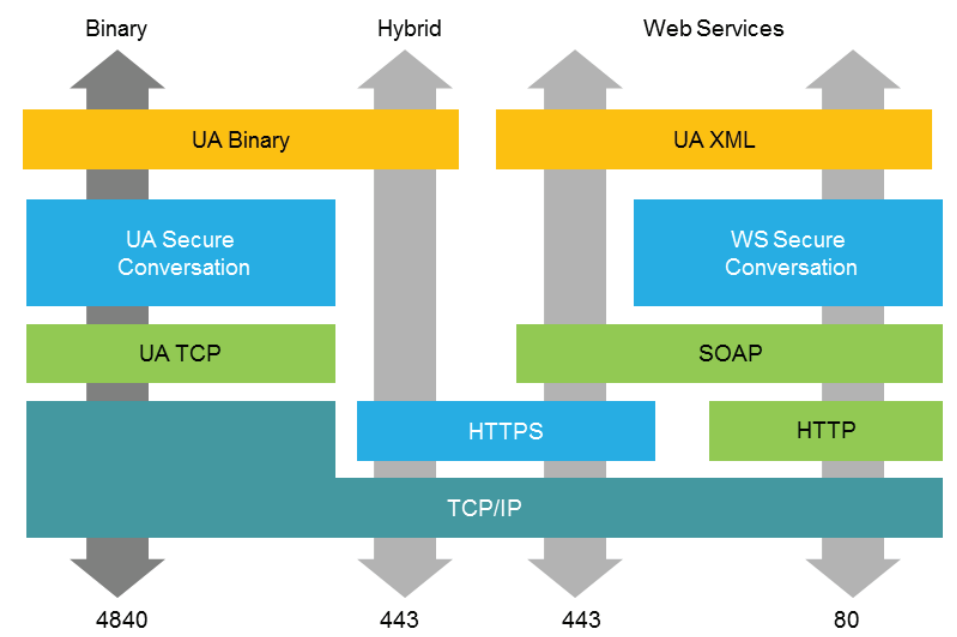

Nota: Prosys OPC, 2013

\section{Protocolo de transporte de OPC UA}

Se trata de un protocolo específico de OPC UA basado en la tecnología TCP, el cual establece un canal full dúplex para transmitir datos binarios entre clientes y servidores OPC-UA. Se diferencia de HTTP porque permite que las respuestas lleguen en cualquier orden, y si la comunicación falla, se permite el uso de puntos finales (Endpoints) de transporte TCP diferentes. Un punto final (Endpoint) en OPC UA debe tener un URL Endpoint, una política de seguridad, un modo de seguridad y el tipo de autenticación. Al referirse a que se pueden utilizar diferentes puntos finales en OPC UA se quiere decir que se puede usar una combinación diferente de estos cuatro campos.

\section{Gestión centralizada}

Mediante una arquitectura cliente-servidor, basada en OPC UA, es posible monitorear en tiempo real los diferentes valores recibidos desde los distintos elementos de automatización, en un mismo punto centralizado, el 
servidor OPC UA es simulado Prosys (Prosys OPC, 2019). Esto garantiza el monitoreo proactivo de la integridad de la información. También el histórico de las informaciones suministradas, está totalmente asegurado y el acceso puede ser restringido, a diferencia de técnicas manuales como se ha mencionado previamente. Tal histórico también puede ser perfectamente centralizado y del mismo modo, puede ser presentado en diferentes formatos según sea conveniente.

Tomando en consideración los elementos técnicos, se verifica que el estándar OPC UA es altamente eficiente, en virtud de que integra cualquier sistema utilizado en la empresa. Así mismo, se resalta que el estándar proporciona buena administración, presentación y seguridad de los datos suministrados por los equipos industriales. Comparable con los resultados obtenidos por Sagñay (2016), que resalta que OPC UA posee todas las características técnicas necesarias para lograr integración industrial de manera segura en entornos multiplataformas.

\section{Arquitectura de redes y comunicaciones con UPC UA}

En el creciente entorno industrial automatizado de Productos del Trópico S.A.S., el esquema actual de gestión de la información está conformado por islas, las cuales generan el manejo de forma manual. Este modelo 'desconectado' provoca inconvenientes en la trazabilidad de la información, además de la gestión proactiva y reactiva ante amenazas en la red (Maddison, 2018).

En el camino hacia la digitalización industrial, el gigante en la industria de la automatización, Siemens (2018), señala a OPC UA como un estándar que permite la interoperabilidad independiente a plataformas y fabricantes, el cual permite implementar un estándar de comunicación capaz de acelerar las transiciones sin comprometer la seguridad de la información.

Por estas razones, y acogidos a las buenas prácticas de la industria, Productos del Trópico S.A.S. implementa una arquitectura unificada (cliente-servidor) basada en OPC UA. Partiendo de esta premisa, el primer paso es conocer la topología actual de redes y comunicaciones en la empresa, para lo cual se utiliza la figura 4. 


\section{Figura 4}

Estructura actual de Productos del Trópico S.A.S.

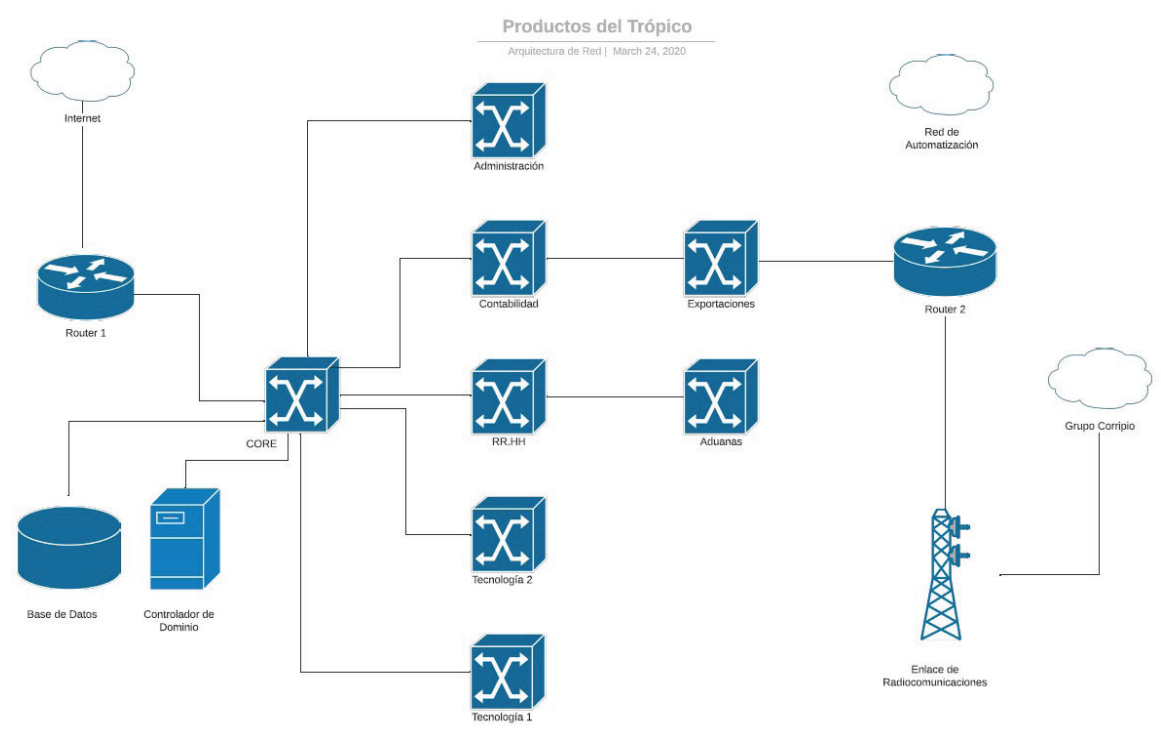

Como se puede visualizar en dicha topología, basados en informaciones sobre su estructura lógica provistas (Monegro, 2020), se pueden resaltar las siguientes mejoras: ausencia de redundancia física y lógica de los Switches de acceso hacia el Switch Core; utilización de la topología Spanning-Tree estándar (802.1D); carencia de LANs virtuales (VLANs) para aislar tráfico de red; falta de administración de los puertos troncales en los Switches; falta de interfaces virtuales conmutadas (SVI) para la administración remota de Switches; poco control entre los roles de los Switches en la arquitectura Spanning-Tree; utilización de instancia única en Spanning-Tree para el tráfico de capa 2; inexistencia de un dominio VTP (Vlan Trunking Protocol); escasez de seguridad remotas y locales en los equipos intermediarios de red (capas 2 y 3 ); ausencia de subredes en el Router principal para habilitar comunicación entre Vlans; aislamiento total de la red de automatización; insuficiencia de ancho de banda en enlaces troncales de segmentos lejanos (latencias); nula existencia de un sistema manejador de eventos (EMS). Dependiendo de la perspectiva, se pueden ańadir elementos adicionales a esta lista para lograr la apropiada arquitectura de redes y comunicaciones que permita el manejo de 
información e integración de sistemas, utilizando el estándar OPC UA en Productos del Trópico S.A.S.

Sin embargo, con el propósito de aminorar los costos de esta propuesta, se han limitado las oportunidades de mejora en los aspectos resueltos de manera lógica (virtual), cuya solución requiere inversiones bajas. Las soluciones para la arquitectura ideal se detallan a continuación:

1. Habilitar la redundancia física y lógica entre los Switches de acceso hacia el Switch Core: se utilizó el software EVE-NG, en el cual se cargan imágenes reales de los sistemas operativos utilizados en los equipos de red. Aquí, se muestra cómo se han adicionado trece (13) enlaces físicos a la topología para garantizar la redundancia entre Switches y, por supuesto, para incorporar la red de automatización a la topología, la cual fue interconectada al servidor OPC UA. El resultado del nuevo esquema propuesto es tolerante a fallos y se evidencia en la figura 5.

\section{Figura 5}

Nueva propuesta de arquitectura redundante

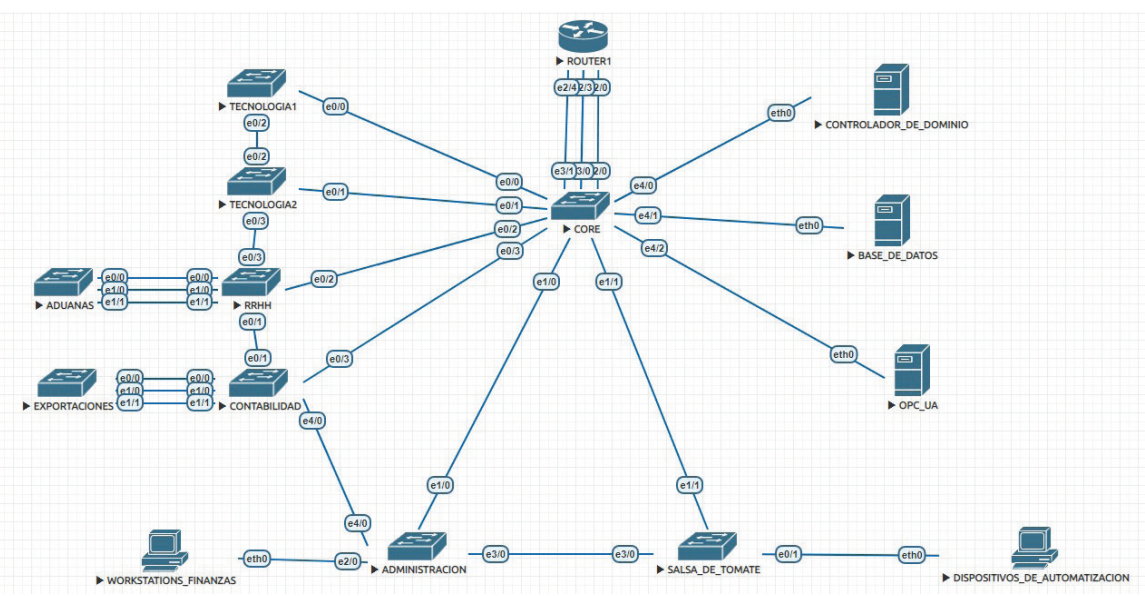

2. Utilizar el protocolo Multiple Spanning Tree Protocol (MSTP) en la topología de Switches: se plantea la utilización de MSTP (Edgeworth et al., 2019), a fin de agrupar múltiples instancias STP en una misma región, disminuyendo ampliamente el tráfico STP y, a su vez, 
habilitando la arquitectura para el balanceo de carga, lo cual mejora la eficiencia de esta en virtud de implementar aplicaciones críticas como OPC UA. Se han agrupado cinco (5) Switches, con el propósito de lograr que desde el punto de vista STP actúen como un solo, esto se muestra en la figura 6 .

\section{Figura 6}

Miembros de la región MST

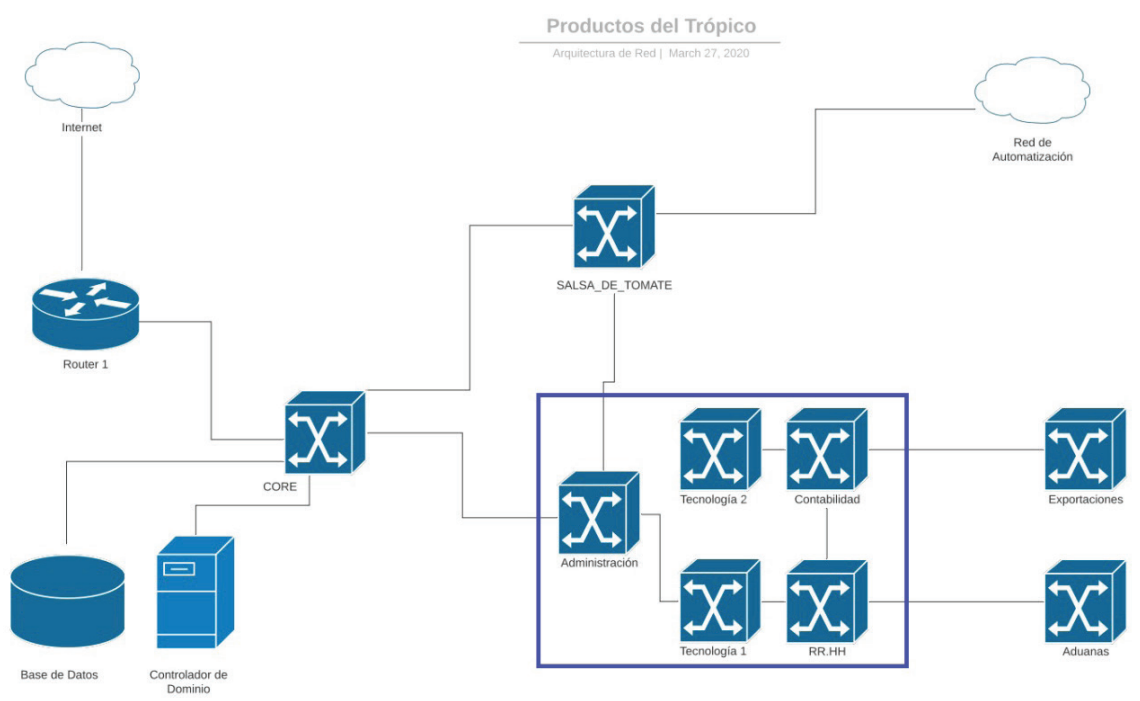

3. Crear LANs virtuales (VLANs) para aislar tráfico de red según sus aplicaciones, disminuyendo las lluvias de broadcast: las Vlans proveen mejor utilización de puertos y permiten que múltiples dominios de broadcast residan en el mismo Switch. Es necesario habilitar este esquema en Productos del Trópico S.A.S. para mejorar el rendimiento de la red a utilizar.

1. Administrar los puertos troncales en los Switches: existen dos (2) formas para el establecimiento de puertos troncales en los Switches: estática y dinámica o automática (Dynamic Trunking Protocol) (Edgeworth et al., 2019). Las buenas prácticas recomiendan el establecimiento estático los puertos troncales para eliminar incertidumbre con relación al estatus de dichos puertos, a fin de aminorar el tiempo en 
procesos de troubleshooting y aportar en la correcta gestión de redes. Se propone la configuración y documentación de puertos troncales en todos los Switches.

2. Crear interfaces virtuales conmutadas (SVI, por su sigla en inglés) para la administración remota de los Switches: los Switches manejan tráfico capa 2 , y es posible crear interfaces virtuales capaces de procesar tráfico de capa 3, a fines de habilitar la administración remota de los Switches, para así gestionar la red y el proceso de troubleshooting en el esquema de conectividad.

3. Controlar los roles de los Switches en la arquitectura Spanning-Tree: Establecer el Switch de mejores prestaciones, de Switch Core, que tenga el rol de root bridge, para garantizar una tolerancia a fallos mucho mayor, minimizar cambios en la topología STP y, por supuesto, una gestión más efectiva de la red. Esta práctica garantiza una colocación consistente del root bridge (Edgeworth et al., 2019).

4. Crear múltiples instancias Spanning-Tree para el tráfico de capa 2: a fin de gestionar apropiadamente todo el tráfico de los Switches, de los cuales se apoya OPC UA, es correcto crear múltiples instancias según las Vlans utilizadas, vía MSTP, ya que permite mapear distintas cantidades de Vlans en un mismo árbol STP. Con esta práctica se garantiza un mejor desempeño de la arquitectura.

5. Establecer un dominio VTP (Vlan Trunking Protocol) para la provisión automatizada de Vlans: con gran cantidad de Switches, como en Productos del Trópico S.A.S., el aprovisionamiento manual para la administración de la red, se recomienda ubicar los Switches en el mismo dominio VTP, con lo que se modifican o borran Vlans en el Switch servidor, y los clientes reciben y replican estas configuraciones.

6. Establecer medidas de seguridad remotas y locales en los equipos intermediarios de red (capa 2 y capa 3): hay muchas formas de promover la seguridad de redes en la topología propuesta. Las formas más elementales son la creación de contraseñas y encriptación en los dispositivos de red para los modos de configuración, el bloqueo de la capacidad de recuperar contraseñas, contraseñas en los accesos 
remotos (líneas vty), y también la aplicación de políticas VRF (Virtual Route Forwarding) en los equipos capa 3.

7. Establecer subredes en el Router principal para habilitar comunicación entre Vlans: si se deseaba habilitar comunicación entre diferentes segmentos de la red, se debía contar con una interfaz física en el Router para cada uno de los referidos segmentos. Sin embargo, en la actualidad esto no es un problema, ya que es posible crear subinterfaces lógicas en los puertos físicos de los Router, lo cual permite y garantiza interconectividad de Vlans sin limitaciones de interfaces físicas. Es necesario resaltar que el servidor OPC UA debe estar en una subred distinta al segmento operativo de la red de automatización.

8. Interconectar la red de automatización: aislar en su totalidad la red de automatización tiene más desventajas que ventajas, ya que no es posible manejar la red desde los sistemas de gestión de la información locales y remotos, lo cual es una tendencia establecida en la industria 4.0; un ejemplo son sistemas que apunta a la virtualización en la nube de elementos pertenecientes a infraestructura de automatización mediante OPC UA (George, 2017). Para ello, es necesario habilitar conexiones desde los dispositivos de automatización hacia los Switches empresariales.

9. Ampliar el ancho de banda en enlaces troncales de los segmentos más alejados en la red a fin de prevenir latencias: los enlaces troncales se pueden saturar y se generan pérdidas de paquetes (Edgeworth et al., 2019), lo que puede generar consecuencias incluso de carácter económico. Afortunadamente, es posible garantizar alta disponibilidad (redundancia) y duplicar (en muchos casos hasta cuadruplicar) el ancho de banda en los enlaces troncales mediante la creación de Etherchannels para estos dispositivos.

10. Implementación de un Sistema Manejador de Eventos (EMS): el propósito principal de esta propuesta es la gestión centralizada, a fines de garantizar la integridad, disponibilidad y seguridad de la información. Sin embargo, se ha detectado que se necesita añadir una gestión efectiva en tiempo real de los eventos ocurridos en la red, de modo que la detección temprana de anomalías pueda prevenir o 
corregir problemas en el proceso; en ese caso se recomienda el EMS Zabbix, véase la figura 7 .

\section{Figura 7}

Gestión de Switch Cisco vía Zabbix

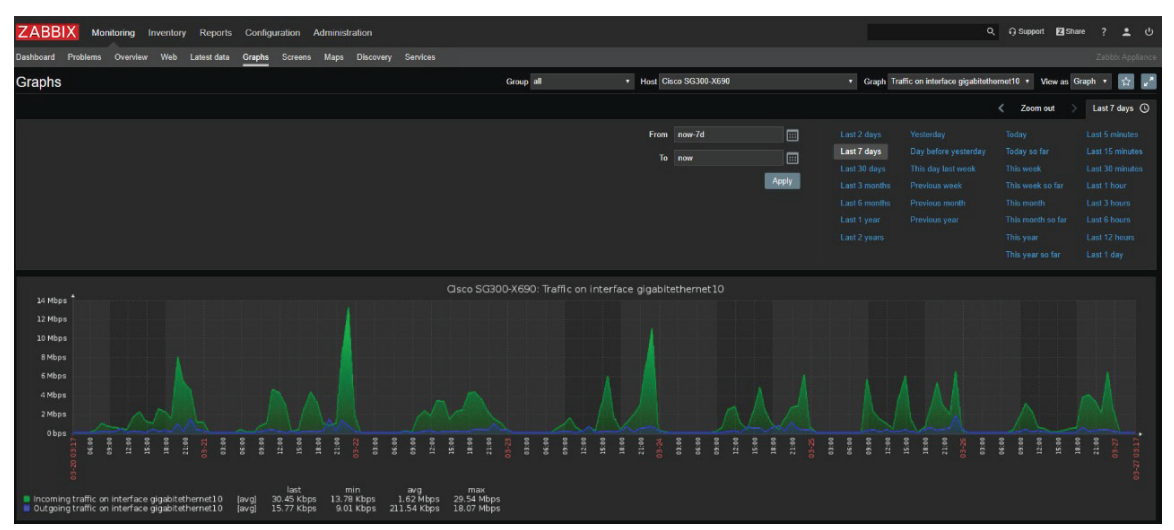

\section{Beneficios de Integración de Flujo de Información con OPC UA en Productos del Trópico S.A.S.}

Dado el alto componente manual en captar y consultar información en la línea Pasta de Tomate, es necesario auditar mensualmente dichas informaciones. Según Monegro (2020), tales auditorías se realizan en promedio dos días durante cada mes del año. Esta información es útil para el determinar el flujo de los procesos y el cálculo de la productividad de los empleados, sobre el cual se realiza el pago de incentivos en la empresa, por tanto, es una variable de gran importancia para todos los involucrados. Las auditorías requieren detener la línea de producción hasta que la misma concluya, de este escenario, se pueden obtener las siguientes conclusiones:

1. De los 365 días del año, en promedio, el proceso de Pasta de Tomate se detiene 24 días (un $6.58 \%$ del tiempo total) con el objetivo de auditar las informaciones obtenidas.

2. Si se consideran los ingresos anuales generados por el proceso Pasta de Tomate como ' $\mathrm{X}$ ', entonces, a causa de estas auditorías la empresa está 
percibiendo una disminución del $6.58 \%$ en sus ingresos obtenidos por dicha línea, o lo que es lo mismo, un 0.0658X.

3. Es evidente que el proceso manual para la generación consulta y auditoría de las informaciones implica que Productos del Trópico S.A.S. tiene oportunidades de mejora en sus políticas de 'continuidad del negocio'.

4. Para validar las informaciones suministradas por la línea 3, si se desea culminar el proceso de forma rápida, se necesita destinar una mayor cantidad de personal para procesar todos los datos, lo cual incide en la inversión de recursos humanos. En caso contrario, si el tiempo de ejecución no es una prioridad, poco personal procesando los datos implica que la información estará lista en un tiempo más prolongado, afectando la disponibilidad de la información para la toma de decisiones operativas y/o administrativas.

Gracias a OPC UA y su versatilidad para integrar el flujo de información entre sistemas es posible superar estas problemáticas de la siguiente manera:

1. Si se implementa la arquitectura unificada (cliente-servidor) basada en OPC UA, la línea de producción puede enviar las informaciones de interés en tiempo real a una base de datos vinculada al ERP, lo cual garantiza continuidad total de los procesos. Esto implica incremento del $6.58 \%$ en sus ingresos por la línea; resultado de no detener la producción por 24 días al año, impactando directamente la continuidad del negocio.

2. Reduce el recurso humano de validación de las informaciones, dada su supervisión en línea de las fuentes de información, que puede ser gestionado de manera local o remota. Lo que implica un uso más eficiente de los recursos humanos y de información.

\section{Conclusiones}

La convergencia entre la estructura de automatización de Productos del Trópico S.A.S con su arquitectura de redes y comunicaciones permite la 
mejora de la producción y rentabilidad de la empresa. La propuesta optimiza la arquitectura informática e interconecta la red de automatización con la red de gestión; acompañado de la virtualización de un firewall y un servidor OPC UA en máquinas virtuales alojadas en cualquiera de los servidores físicos, con lo cual la empresa estará preparada para aplicar cualquier tendencia de la Industria 4.0. y del mismo modo reducir los riesgos y el impacto económico de tener islas de información (Maddison, 2018).

Una arquitectura unificada (cliente-servidor) es capaz de gestionar y centralizar la información; en primera instancia garantiza la continuidad del negocio, acarreando consigo mayor rentabilidad financiera, con un crecimiento del $6.58 \%$. Solo por la disminución de las demoras por paros de procesos productivos periódicos de casi el $7 \%$ del ciclo anual. Del mismo modo, tal arquitectura promueve un control eficaz de los procesos y estadísticas claves para el crecimiento sostenible de Productos del Trópico S.A.S., y, también, garantiza que las informaciones obtenidas de los diferentes elementos industriales podrán estar salvaguardadas contra cualquier tipo de intervención humana y/o artificial.

Tomando como referencia las problemáticas descritas que actualmente afectan a la empresa, así como también la necesidad generalizada de utilizar cinco (5) protocolos y/o estándares diferentes propios de cada marca para compartir información con equipos de otros fabricantes, es altamente recomendable la implementación de una arquitectura unificada (cliente-servidor) basada en OPC UA para eliminar la necesidad de contar con programas de intercomunicación (drivers) específicos entre cada aplicación y el origen de los datos. Esto nos permite reducir enormemente la necesidad de drivers entre los dispositivos industriales en un $80 \%$. Del mismo modo, permite crear los medios necesarios para administrar y/o acceder a las informaciones de carácter crítico, provenientes de las distintas líneas de producción, según las necesidades actuales del negocio.

Conforme al desarrollador de servidores OPC UA (Prosys OPC, 2019), varias empresas han optado por eficientizar sus procesos y mejorar el flujo de información de estos utilizando el estándar OPC UA. Según esta entidad, el $25 \%$ de las empresas a las cuales les han brindado servicios están dedicadas a la producción de alimentos y las mismas han experimentado 
mejoras sustanciales en sus procesos productivos. Esta es la situación que se pretende alcanzar en Productos del Trópico S.A.S. a raíz de la propuesta de implementación del referido estándar. Es importante recordar y señalar que esta arquitectura propuesta plantea un mejor uso de los recursos existentes, más atractivo para el usuario final. La arquitectura unificada basada en OPC UA es la solución en términos costo/efectividad, según indican miembros del consejo de directores de OPC Foundation, como son Microsoft, SAP, Schneider Electric, Rockwell Automation, Siemens, Honeywell, entre otros (OPC Foundation, s.f.).

\section{Reconocimientos}

Esta investigación se da como resultado de la colaboración de las dos universidades de alto prestigio en Venezuela en el área de automatización, en conjunto con el Instituto Especializado de Estudios Superiores Loyola, con la finalidad de establecer lazos académicos que permitan un crecimiento en el área de investigación y aplicación del conocimiento de la Industria 4.0 al entorno dominicano.

El trabajo no recibió financiamiento, ya que es producto de una investigación colaborativa.

\section{Referencias}

Baño, H. F. (2019). Sistema empotrado de bajo costo para adquisición de datos basado en el estándar de comunicación industrial OPC-UA. Ambato: Universidad Técnica de Ambato.

Barnstedt, E. (2019). Interoperability for Industries 4.0 And the Internet of Things. OPC Foundation, 56.

Edgeworth, B., Garza Rios, R., Hucaby, D., \& Gooley, J. (2019). CCNP and CCIE Enterprise Core ENCOR 350-401 Official Cert Guide. California: Cisco Press.

EU Automation. (15 de mayo de 2019). AUTOMATED. Obtenido de Eu Automation: https://www.euautomation.com/es/automated/ article/opc-ua-en-la-fabrica-inteligente

Felt, A. (2019). Interoperability for industries 4.0 and the Internet of things. OPC Foundation, 56. 
Garay, C. (Enero de 2020). Entrevista Producto del Tropico. (W. Emeterio, Entrevistador)

George, S. (18 de septiembre de 2017). Simplifying OPC UA security for everyone. Obtenido de https://azure.microsoft.com/es-es/blog/ simplifying-opc-ua-security-for-everyone/

INTRAVIS. (2017). INTRAVIS. Obtenido de INTRAVIS: https://www. intravis.de/es/industria-40/case-studies/

Maddison, J. (5 de septiembre de 2018). Fighting Network Silos. Obtenido de https://www.fortinet.com/blog/business-and-technology/fightingnetwork-siloes.html

Molina, U. G. (8 de febrero de 2019). HoyDigital. Obtenido de Hoy: https://hoy.com.do/empresa-productos-del-tropico-inauguraplanta-de-tratamiento-aguas-residuales-en-sc/

Monegro, J. C. (16 de enero de 2020). Entrevista Productos del Trópico. (W. Emeterio, Entrevistador)

OPC Foundation. (s.f.). Organization of OPC Foundation. Recuperado el 15 de diciembre de 2019, de https://opcfoundation.org/about/ opc-foundation/organization/

Prosys OPC. (20 de diciembre de 2013). OPC UA 1.02. Obtenido de https://www.prosysopc.com/blog/opc-ua-1-02/

Resnick, C., \& Clayton, D. (2018). OPC Technology Well-positioned for Further Growth in Tomorrow's Connected World. Dedham: ARC.

Ruiz, M. (15 de noviembre de 2019). Charla de Seguridad Corporativa. (E. Gabriel, Entrevistador)

Sagñay, F. J. (2016). Estudio Comparativo mediante simulación de las variantes de la tecnología OPC-UA y su utilización en controladores industriales. Quito: Pontificia Universidad Católica del Ecuador. 